\title{
A conformational transition in the adenylyl cyclase catalytic site yields different binding modes for ribosyl-modified and unmodified nucleotide inhibitors
}

\author{
Jenna L. Wang, ${ }^{\mathrm{a}, \dagger}$ Jian-Xin Guo,,${ }^{\mathrm{a}, \mathrm{b}, \dagger}$ Qi-Yuan Zhang, ${ }^{\mathrm{c}}$ Jay J.-Q. Wu, \\ Roland Seifert ${ }^{\mathrm{d}}$ and Gerald H. Lushington ${ }^{\mathrm{a}, *}$ \\ ${ }^{a}$ Molecular Graphics and Modeling Laboratory, University of Kansas, Lawrence, KS 66045, USA \\ ${ }^{\mathrm{b}}$ VM Discovery, Fremont, CA 94538, USA \\ ${ }^{\mathrm{c}}$ State Key Laboratory for Structural Chemistry of Stable \& Unstable Species, Institute of Chemistry, \\ Chinese Academy of Sciences, Beijing, PR China \\ ${ }^{\mathrm{d}}$ Department of Pharmacology and Toxicology, University of Regensburg, Germany
}

Received 16 October 2006; revised 2 February 2007; accepted 8 February 2007

Available online 11 February 2007

\begin{abstract}
Adenylyl cyclases (ACs) are promising pharmacological targets for treating heart failure, cancer, and psychosis. Ribosesubstituted nucleotides have been reported as a potent family of AC inhibitors. In silico analysis of the docked conformers of such nucleotides in AC permits assembly of a consistent, intuitive QSAR model with strong correlation relative to measured $\mathrm{p} K_{\mathrm{i}}$ values. Energy decomposition suggests that the MANT group effects an AC conformational transition upon ligand binding.

(C) 2007 Elsevier Ltd. All rights reserved.
\end{abstract}

\section{Introduction}

The ubiquitous second messenger cyclic adenosine $3^{\prime}, 5^{\prime}$-monophosphate (cAMP) plays a key role in the intracellular signaling pathways of hormones, neurotransmitters, odorants, and autacoids. ${ }^{1}$ The synthesis of cAMP from ATP is catalyzed by adenylyl cyclase (AC) enzymes. Mammalian adenylyl cyclases (mACs) are a family of integral membrane proteins with at least nine isoforms, each regulated in a unique fashion by association with the stimulatory $G$ protein $\alpha$ subunit $\left(\mathrm{G} \alpha_{\mathrm{s}}\right)$, cofactor binding of the diterpene forskolin (FSK) and its soluble derivatives (except for type IX), exposure to $\mathrm{Ca}^{2+}$, and protein phosphorylation. ${ }^{2}$ In addition to evidence suggesting correlations between elevated $\mathrm{AC}$ activity and contractile dysfunction in human heart failure, ${ }^{3}$ suppressing the function of $\mathrm{AC}$ isoform 5 (i.e., AC5) has been found to provide substantial protection against myocardial apoptosis arising from cardiac

\footnotetext{
Keywords: Adenylyl cyclase; Conformational transition; Molecular modeling; MANT-substituted nucleotides.

* Corresponding author. Tel.: +1 785864 1140; fax: +1 785864 5326; e-mail: glushington@ku.edu

$\dagger$ These authors contributed equally to this work.
}

pressure overload in a mouse model. ${ }^{4}$ Both effects imply the potential importance of $\mathrm{AC}$ inhibition as a mode of preventing heart failure. AC inhibitors may also be potentially used for treatment of cancer, ${ }^{5}$ psychosis, ${ }^{6}$ and for the purposes of contraception or treatment of male infertility. 7,8

ACs contain 12 transmembrane helices, and two intracellular lobes generally referred to as $C_{1}$ and $C_{2}$. The nucleotide-binding site of the protein consists of two homologous cytoplasmic domains, $\mathrm{C}_{1 \mathrm{a}}$ and $\mathrm{C}_{2 \mathrm{a}}$, within the intracellular lobes. These two domains are comprised of approximately 230 amino acids that share at least $50 \%$ similarity across the AC family and are structurally similar. ${ }^{9}$ Previous mutagenesis analysis revealed that residues from both $\mathrm{C}_{1 \mathrm{a}}$ and $\mathrm{C}_{2 \mathrm{a}}$ domains contribute to ATP binding and catalysis. ${ }^{10,11}$ Recent X-ray crystal structures of the catalytic site ${ }^{9,12}$ provide further insight into AC catalysis and inhibition, collectively illuminating a receptor that can be characterized as having three main features: (1) a phosphate binding region containing two metal ions (typically $\mathrm{Mg}^{2+}$ or $\mathrm{Mn}^{2+}$ ), (2) a purine ring binding site possessing $\mathrm{H}$-bond donating and accepting side-chains suitably oriented for affixing adenosine, and (3) a hydrophobic pocket. 
From available X-ray crystallographic structures, ${ }^{1,7,13}$ it is apparent that ACs exist in at least three conformational states: (1) a catalytically inactive state, (2) an active state unbound by activators such as forskolin and $\mathrm{G} \alpha_{\mathrm{s}}$, and (3) an active substrate-bound state. In addition to chain flexibility, key structural differences arise from the relative orientation of the associated $\mathrm{C}_{1}$ and $\mathrm{C}_{2}$ lobes. Comparing the relative $\mathrm{C}_{1} / \mathrm{C}_{2}$ orientation within a forskolin-bound $\mathrm{C}_{1} \cdot \mathrm{C}_{2}$ heterodimer on one hand, with the $\mathrm{C}_{2} / \mathrm{C}_{2}$ orientation in a $\mathrm{C}_{2} \cdot \mathrm{C}_{2}$ homodimer, one observes a $7^{\circ}$ inter-lobe rotational difference. The rotational state of the former brings key catalytic elements from the two domains about $2 \AA$ closer to each other. It is reasonable to conclude that in each of the above cases at least some of the observed structure differences arise from AC interactions with forskolin and G-protein subunits, and that these interactions thus dynamically alter binding mechanics within the nucleotide receptor.

Many inhibitors to the AC nucleotide receptor, including numerous nucleotides and nucleotide analogs, also induce AC structural relaxation. So-called p-site inhibitors exhibit non-competitive inhibition by initiating a transition in the $\mathrm{C}_{1} \cdot \mathrm{C}_{2}$ moiety from an 'open' to a 'closed' conformation. This transition entails the $\alpha 1-$ $\alpha 2, \beta 2-\beta 3$, and $\alpha 3-\beta 4$ loops of $C_{1}$ and the $\beta 7^{\prime}-\beta 8^{\prime}$ loop of $\mathrm{C}_{2}$ moving toward each other, thus closing the gap between the purine-binding pocket in the $\mathrm{C}_{2}$ domain and the triphosphate binding loop (P-loop) located primarily in the $\mathrm{C}_{1}$ domain. ${ }^{9,14}$ Recently, we have identified novel competitive nucleotide AC inhibitors with $N$-methyl-anthranoyl (MANT) substituents on ribosyl hydroxy oxygens. ${ }^{15} \mathrm{~A}$ crystal structure of the AC complex with MANT-GTP $\left(K_{\mathrm{i}}=4.2 \mathrm{nM}\right)$ has revealed that the MANT group binds to a conserved hydrophobic patch near the center of the cavity, and prevents mACs from undergoing the 'open' to 'closed' transition. ${ }^{14,16}$ In the present paper, we will focus on the characterization of the purine and pyrimidine MANT-nucleotide inhibition mode, determining whether it, too, may induce structural relaxation effects in the receptor, and identifying trends favoring potent $\mathrm{AC}$ inhibition.

\section{Results and discussion}

\subsection{Ribosyl-substituted nucleotides}

MANT-GTP is one of the most potent known AC inhibitors, binding to the nucleotide binding site with a $K_{\mathrm{i}}$ of $4.2 \mathrm{nM} .^{16}$ While the MANT group, on average, enhances inhibitive potency relative to NRM nucleotides, ${ }^{15}$ substantial variations in affinity also occur as a function of the purine/pyrimidine rings and modifications to the phosphate tail. To explore structure-activity relationships among MANT-nucleotide inhibitors of AC, we applied our modified COMBINE approach to the prediction of bound state conformers, $K_{\mathrm{i}}$-values, and interaction coefficients for the set of ligands listed in Table 1. In doing so, we were able to achieve bound conformers whose interactions were reasonably consistent with experimental $K_{\mathrm{i}}$-values for 21 of the 23 ligands in the test set, with only ITP $\gamma \mathrm{S}$ and MANT-XDP failing to yield a plausible docked conformer. The most probable explanations for these outlier cases include either error in the experimentally determined affinity or possible post-complexation relaxation effects that are not well reflected in the 1TL7 crystal structure used as a receptor model. The set of bound ligand conformers used in the

Table 1. Comparison of predicted inhibition constants for our training set of AC inhibitors versus previously published experimental data as measured in excess $\mathrm{Mn}^{2+16}$

\begin{tabular}{|c|c|c|c|c|}
\hline Nucleotide & $K_{\mathrm{i}}$ (nM; expt.) & $\mathrm{p} K_{\mathrm{i}}$ (expt.) & $\mathrm{p} K_{\mathrm{i}}$ (pred.) & Predicted ribose substitution \\
\hline MANT-GTP & 4.2 & 8.38 & 7.27 & $3^{\prime}$ \\
\hline MANT-ITP $\gamma$ S & 19 & 7.72 & 8.04 & $3^{\prime}$ \\
\hline MANT-AppNHp & 20 & 7.70 & 7.20 & $2^{\prime}$ \\
\hline MANT-GTP $\gamma$ S & 24 & 7.62 & 7.27 & $3^{\prime}$ \\
\hline MANT-GppNHp & 34 & 7.47 & 8.04 & $3^{\prime}$ \\
\hline MANT-ATP & 35 & 7.46 & 6.80 & $3^{\prime}$ \\
\hline 2'-d-3'-MANT-GTP & 45 & 7.35 & 6.58 & \\
\hline MANT-ADP & 57 & 7.24 & 7.46 & $3^{\prime}$ \\
\hline $2^{\prime}-\mathrm{d}-3^{\prime}-\mathrm{MANT}-\mathrm{GppNHp}$ & 200 & 6.70 & 7.27 & \\
\hline MANT-GDP & 290 & 6.54 & 7.27 & $3^{\prime}$ \\
\hline MANT-XppNHp & 330 & 6.48 & 6.68 & $3^{\prime}$ \\
\hline $2^{\prime}$-d-3'-MANT-ATP & 460 & 6.34 & 6.56 & \\
\hline ITP $\gamma \mathrm{S}$ & 1200 & 5.92 & - & \\
\hline BODIPY-FL-GppNHp & 1400 & 5.85 & 5.87 & $2^{\prime}$ \\
\hline $2^{\prime}-\mathrm{d}-3^{\prime}-\mathrm{MANT}-\mathrm{GDP}$ & 3400 & 5.47 & 5.24 & \\
\hline MANT-XDP & 5800 & 5.24 & - & \\
\hline $\mathrm{UTP} \gamma \mathrm{S}$ & 8500 & 5.07 & 4.95 & \\
\hline 6-MAH-cAMP & 43,000 & 4.37 & 4.29 & \\
\hline BODIPY-FL-GTP $\gamma \mathrm{S}$ & 79,000 & 4.10 & 4.24 & $2^{\prime}$ \\
\hline $2^{\prime}$-d-UTP & 100,000 & 4.00 & 4.91 & \\
\hline MANT-cAMP & 100,000 & 4.00 & 3.94 & \\
\hline MANT-cGMP & 100,000 & 4.00 & 4.02 & \\
\hline UTP & 100,000 & 4.00 & 3.96 & \\
\hline
\end{tabular}

$\mathrm{p} K_{\mathrm{i}}=9-\log _{10}\left(K_{\mathrm{i}}\right)$. 
construction of our COMBINE model is provided as Supplementary material. These conformers correspond well to experimental data, with a trained three-component PLS fit displaying strong correlation $\left(R^{2}=0.89\right)$ and a reasonable leave-one-out cross-validation $\left(Q^{2}=0.61\right)$ score. It should be noted that coefficients on the desolvation terms $\triangle P S A$ and $\triangle N P S A$ were found to be very small and that their omission yielded a model with nearly identical behavior relative to the first, with $R^{2}$ and $Q^{2}$ values and most $\mathrm{p} K_{\mathrm{i}}$ predictions only varying in the second or third decimal places (Fig. 1).

In order to provide an independent test of model predictivity, a series of seven trinitrophenol (TNP) substituted nucleotide analogs (TNP-ATP, TNP-ADP, TNP-AMP, TNP-GTP, TNP-GDP, TNP-UTP, and TNP-CTP, respectively) and one MANT-substituted species with an aminoethyl-carbamoyl (EDA) linker to the ribosyl (MANT-EDA-ATP), whose structures and AC inhibition activity are reported in recent work by Mou et al. were studied. ${ }^{17}$ Our calculations attained a strong degree of qualitative correlation with the experimental data ( $R^{2}=0.76$; see Fig. 2 ), accurately reproducing the general activity trend. From Figure 2, one does observe a consistent trend by our method toward overestimating the affinity of these test set molecules.

From the COMBINE training process, we have ascertained that the $3^{\prime}$-ribose substituents are preferred over the $2^{\prime}$-analogs in the majority of cases. Of the 11 ligands for which autoconversion is possible between ribose substitution points, eight were found to adopt the $3^{\prime}$-substitution form and only MANT-AppNHp and the two BODIPY species are predicted to retain a $2^{\prime}$-substituent.

In prior pharmacophoric studies of the AC system, a number of receptor residues have been qualitatively identified as providing features for interaction with observed inhibitors. These include: Gly 399, Phe 400, Thr

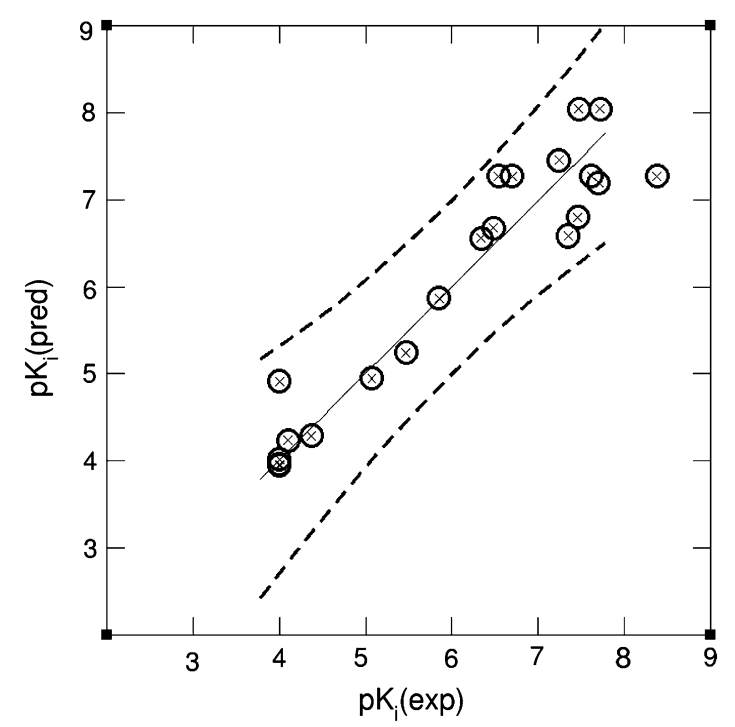

Figure 1. Correlation between experimental and predicted $\mathrm{p} K_{\mathrm{i}}$ values for ribosyl-substituted nucleotides interacting with $\mathrm{Mn}^{2+}$ enriched AC. Best fit linear regression curve is shown (solid line; $R^{2}=0.89$; $Q^{2}=0.61$ ), plus $95 \%$ confidence intervals.

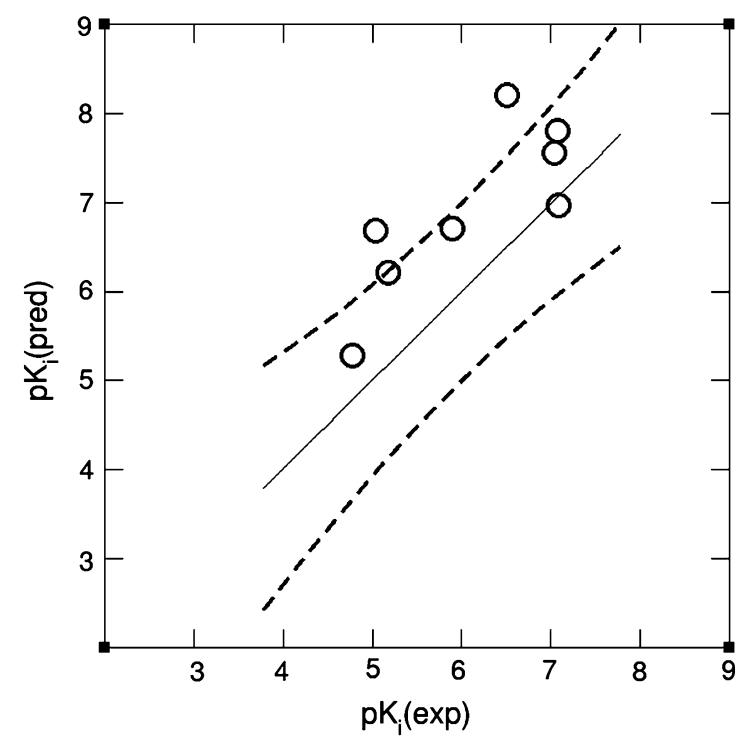

Figure 2. Predicted values for our eight-compound test set composed of 7 TNP-nucleotides, plus MANT-EDA-ATP, plotted relative to experimental values. These predictions are overlaid with the correlation curve and $95 \%$ confidence interval derived from the training set (see Fig. 1), revealing that the bioactivity trend for the test set predictions correlates well qualitatively with that of the training set, but tends to overestimate bioactivity.

401, Leu 438, and Asp 440 (all $\mathrm{C}_{1}$ constituents), as well as Lys 938, Asp 1018, Leu 1019, Gly 1021, Asn 1022, Asn 1025, Ser 1028, Arg 1029, and Leu 1065 (all $\left.\mathrm{C}_{2}\right) \cdot{ }^{9-16,18}$ COMBINE analysis allows one to augment such intuitive insight by exposing other residues that, although possibly exerting smaller average effects on ligand affinity, may discriminate between strong and weak ligands more effectively than the above highly conserved interactions, thus providing new avenues for inhibitor refinement. To this end, those residues with optimal tendencies toward ligand discrimination (as judged by absolute magnitude of the PLS coefficient and relative proximity to the $\mathrm{P}$-site) are indicated in Figure 3 as a function of their COMBINE coefficients, and their corresponding interaction enthalpy $(E)$ and coefficient $* E$ information are reported in Table 2 . As a complement to this, a depiction of the average pharmacophore arising from the full set of 21 bound ligand conformers is provided in Figure 4 against the backdrop of those discriminatory residues identified in Figure 3. Figure 4 provides a clear qualitative depiction of the three-point pharmacophore model recently described for AC inhibition, ${ }^{19}$ wherein the ligand binding mode is dominated by three main features: (1) a triphosphate binding region rendered as a predominantly red (anionic) lobe at the bottom of the graphic, (2) a mixed red/ blue (H-donor/H-acceptor) polar region in the inner region of the top part of the graphic corresponding to the purine base binding site, and (3) the predominantly yellow hydrophobic pocket (outer top region). The interactions corresponding to these pharmacophore elements are described in the succeeding subsections.

2.1.1. Triphosphate binding region. The phosphate tails of these nucleotide ligands interact primarily with two 


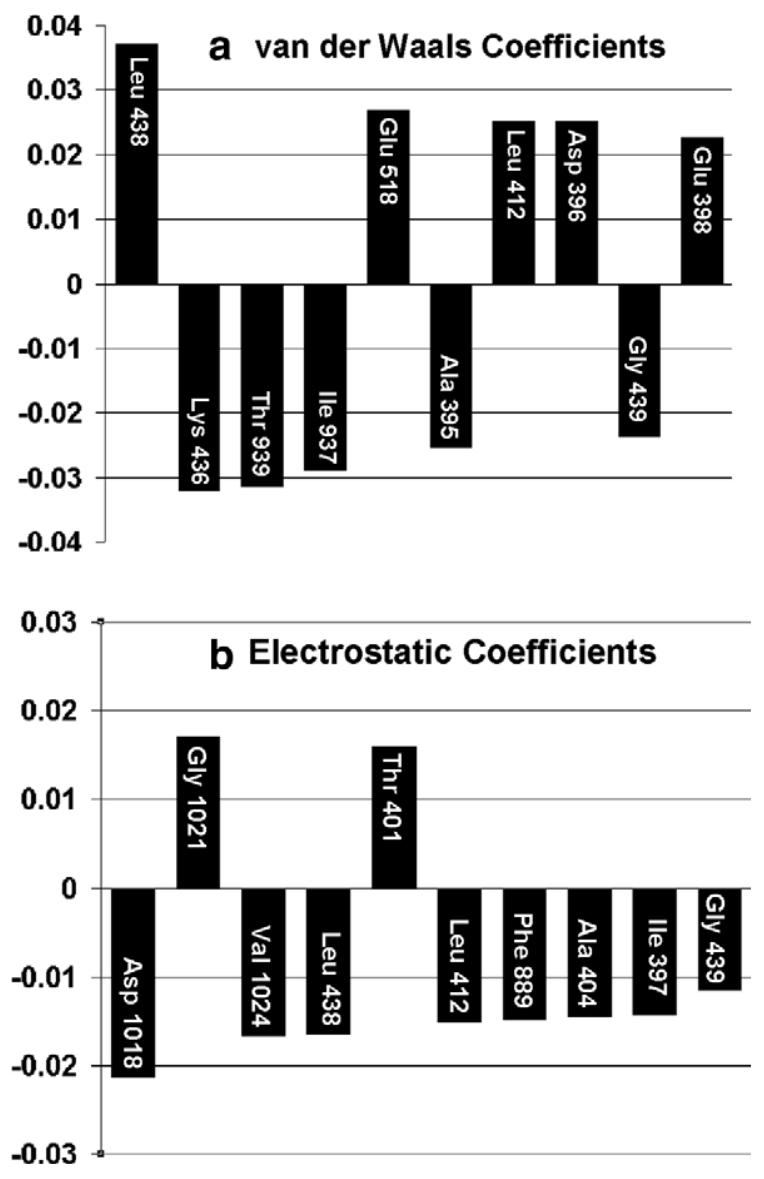

Figure 3. COMBINE coefficients for the ten residues of greatest importance to discriminating ligand binding affinities as a function of van der Waals (a) and electrostatic (b) interactions. Selected residues are those residues within $8 \AA$ of the ligand binding site that possess the ten largest COMBINE coefficients. The signs of coefficients characterize whether the observed affinity trend correlates (negative sign) or anticorrelates (positive) with a specific ligand-residue interaction. Large coefficient magnitude results when the COMBINE model accentuates the role of a given interaction in distriminating binding affinity.

receptor metal ions, whereby oxygens on the head and middle phosphates ( $\alpha$ and $\beta$ ) electrostatically couple with metal A, while the terminal $(\gamma)$ phosphate of triphosphate ligands coordinates with metal $\mathrm{B}$. The strong triphosphate binding affinity relative to di- and mono- phosphates may result from a very favorable binding environment involving ionic interactions with $\mathrm{NH}_{3}{ }^{+}$of Lys 1065, and hydrogen bond donor sites from the backbone NHs of the Thr 401 and Phe 400. Of these three features, only Thr 401 is listed as one of the most important discriminatory residues according to COMBINE coefficients, which implies that the others support interactions that are relatively uniformly conserved among the ligand manifold. Instead, in addition to Thr 401, the residues that appear to influence selectivity are Ala 395, Asp 396, Ile 397, Glu 398, and Glu 518. Among these, Ile 397 has a clear significance: its large negative electrostatic coefficient indicates that it is an unfavorable interaction site for negatively charged ligands largely because it orients a backbone carbonyl toward the phosphate binding site, and its large negative VDW coefficient implies that it provides an unfavorable surface for hydrophobic interactions. Thus, any ligand that approaches close to Ile 397 (as might be the case for species with bulky groups attached to the purine or ribose) is expected to be a poor AC inhibitor. The nearby Ala 395 is also unfavorable for non-polar interactions.

Interestingly, the three anionic residues identified above from COMBINE analysis (Asp 396, Glu 398, and Glu 518 ) actually derive their importance from VDW interactions rather than electrostatics. This correlation is likely somewhat artificial: the main enzymatic role of these residues is in inducing the receptor cations (the two metal ions and Lys 1065) to be positioned favorably for attracting the anionic phosphate tail. A ligand binding mode close to these cations thus corresponds to sufficient proximity to the receptor's partner anions to register a 'favorable' van der Waals interaction, which is plausible since van der Waals interactions generally have a longer-range influence than electrostatics, as has been reported elsewhere for metastable ion-ion attractions. $^{20}$

2.1.2. Nucleotide base binding site. The purine ring binding site is mainly hydrophobic, but does support $\mathrm{H}$-bonding or electrostatic interactions capable of distinguishing among different purine or pyrimidine rings. Immediately adjacent to the purine or pyrimidine rings is a surface formed by exposed backbones of Leu 438 and Gly 439 providing complementary electrostatic

Table 2. Ligand-residue coefficients, interaction enthalpies $(E)$, and products of the coefficient and $E$ for those important residues identified in Figure 3

\begin{tabular}{lrcclrrr}
\hline Interaction & Coeff. & $E(\mathrm{vdW})$ & Coeff. $* E$ & Interaction & \multicolumn{1}{c}{ Coeff. } & $E$ (ele) & Coeff. $* E$ \\
\hline Leu 438 & 0.037 & 6.805 & 0.253 & Asp 1018 & -0.021 & 4.930 \\
Lys 436 & -0.032 & -0.060 & 0.002 & Gly 1021 & 0.017 & -0.105 \\
Thr 939 & -0.031 & -0.049 & 0.002 & Val 1024 & -0.017 & -0.365 \\
Ile 937 & -0.013 & -0.114 & 0.001 & Leu 438 & -0.016 & 0.006 \\
Glu 518 & 0.027 & 1.421 & 0.038 & Thr 401 & 0.016 & 1.178 & -0.005 \\
Ala 395 & -0.025 & -0.058 & 0.001 & Leu 412 & -0.015 & -0.179 \\
Leu 412 & 0.025 & -0.032 & -0.001 & Phe 889 & -0.015 & -0.075 \\
Asp 396 & 0.025 & 4.787 & 0.120 & Ala 404 & -0.015 & -0.546 & 0.025 \\
Gly 439 & -0.024 & 1.697 & -0.040 & Ile 397 & -0.014 & 6.462 & 0.001 \\
Glu 398 & 0.023 & -0.301 & -0.007 & Gly 439 & -0.012 & -0.939 & -0.092 \\
\hline
\end{tabular}

$\Delta \mathrm{H}$ corresponds to the average enthalpy over the full set of training set ligands. 


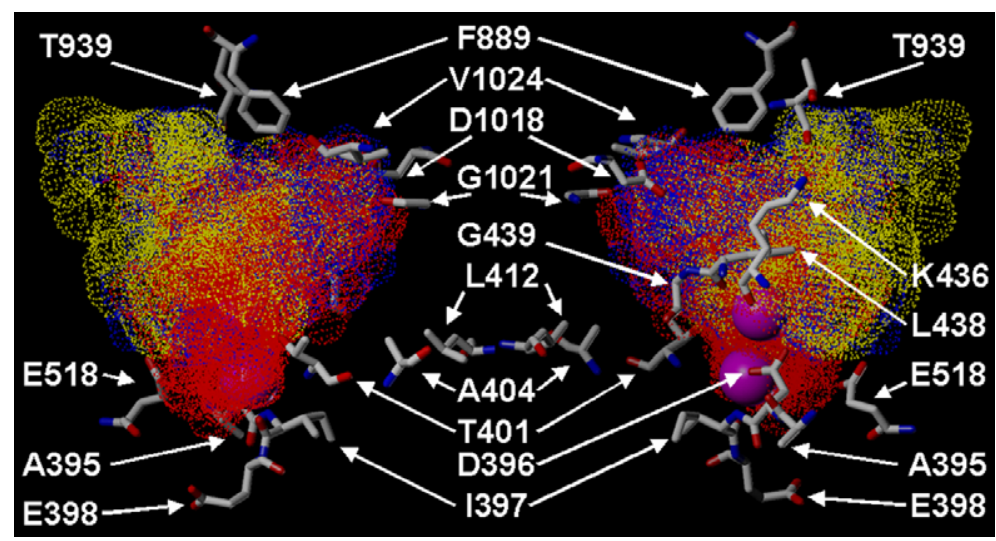

Figure 4. Front (left side) and back (right side) views of the cumulative pharmacophore resulting from all 21 ribose-modified AC inhibitors superimposed in their predicted docked conformers within the framework of the AC receptor. Each ligand is rendered as a dotted Connolly surface, colored to reflect hydrophobic surfaces (yellow), polar electropositive (blue), and polar electronegative (red). Key receptor residues, as defined by close proximity to the binding site and by larger COMBINE coefficients, are rendered in stick form and colored according to the standard CPK scheme.

interactions. While most of the residues interacting with the guanine ring in bound MANT-GTP do so via VDW interactions, the base does form H-bonds to Lys 938, Asp 1018 and Ile 1019. Thr 939 does not have a direct contact with the purine, but appears to exert a substantial non-local influence as gauged by its substantial COMBINE coefficient in Figure 3. One may surmise that ATP analogs have substantially weaker interactions with the purine subsite than do GTP analogs. This would be a reasonable behavior for a substrate such as ATP that, catalytically, should be expected to have only modest barriers to receptor evacuation as the cAMP product. This would also explain observations of substantially greater affinity for MANT-GTP and GTP $\gamma$ S relative to MANT-ATP and ATP $\gamma \mathrm{S} .{ }^{16,18,21}$ They do not, however, explain why the adenine analogs of other, non-triphosphate, systems (e.g., AppNHp, MANT-AppNHp, MANT-ADP) are frequently more potent inhibitors than their guanine analogs. Our simulations suggest, however, that in some cases the adenine ring may be energetically most favorable when rotated $180^{\circ}$ relative to the conformation observed crystallographically for MANT-ATP. ${ }^{14}$ In doing so, the ring recoups $\mathrm{H}$-bonds with Asp 1018, and Ile 1019 (although not with Lys 938) that could effectively negate most of the affinity advantage otherwise observed for guanine analogs. While our docking studies do suggest that this flipped adenine ring is feasible even for MANT-ATP, it may be most evident for ligands with less optimal anionic tail groups (i.e., nontriphosphates). The potential benefits of this alternative orientation may be further augmented in the case of MANT-AppNHp by the possible positioning of the MANT at the $2^{\prime}$ position on the ribose.

Among the other residues within the purine binding pocket identified as potentially important via our COMBINE analysis, most appear to influence primarily via electrostatics, where the Gly 1021 carbonyl is considered a favorable target for electrostatic coupling with ligand electropositive groups, and the backbone amide protons of Gly 439 and Val 1024 appear to be attractive to elec- tronegative species. Phe 889 does not orient its backbone toward the purine, thus one may surmise that the fairly large electronegative COMBINE coefficient is a spurious product of noise in the model.

2.1.3. Hydrophobic pocket. This pocket is an important region for considering ligand modifications for the purpose of optimizing inhibitor potency, with numerous hydrophobic VDW contacts potentially available in this area, the most important of which are likely Ala 409, Asn 1022, Gly 1021, Trp 1020, Val 413, Leu 412, Ala 404 , and Phe 400 . Whereas prior modeling studies ${ }^{15,16}$ have suggested that the $2^{\prime}$-MANT is more favorable than $3^{\prime}$-MANT position, our COMBINE analysis herein, as well as recent crystallographic data ${ }^{14}$ and the 10 -fold poorer affinity of $2^{\prime}$-d-3'-MANT-GTP $\left(K_{i}, 45 \mathrm{nM}\right)$ relative to MANT-GTP $\left(K_{i}, 4.2 \mathrm{nM}\right)$, all argue for the MANT to be in the $3^{\prime}$ position. This preference appears to result from a receptor conformational change, as evidenced by comparisons of crystal structures for the AC/ATP $\alpha \mathrm{S}$ and AC/MANT-GTP complexes.

Reliable COMBINE-based ranking of specific pharmacophoric elements in the hydrophobic pocket is complicated by the fact that coefficients for these residues are comparatively small. This arises primarily from poor conservation of the ligand binding mode in this pocket. Specifically, variations within the rest of the molecule, such as the length or chemical constitution of the phosphate tail, location of ribose modification, and the presence or absence of monophosphate cyclization and partial deoxification of the ribose, all seem to have substantial impact on the orientation of the hydrophobic ribose substituent, even when that substituent (such as MANT) is conserved across a set of ligands. More intuitive detail would be available from a COMBINE model derived by training to inhibition data for a ligand set wherein the primary source of diversity arises from varied ribose substitution, while the phosphate tail and purine structures were kept largely consistent. Unfortunately, no such data set of statistically tenable size is yet available. 


\subsection{Non-ribosyl-modified nucleotides}

In previous $\mathrm{AC}$ inhibition analysis we performed similar docking and COMBINE analysis on a set of NRM purine and pyrimidine nucleotides that varied as a function of modifications on the base or the phosphate. ${ }^{18}$ The resulting bound conformers for the 17 species studied therein provide useful points of comparison relative to the ribosyl-substituted analogs and are thus depicted in Figure 5. These ligands occupy the ATP binding site with the phosphate tail coordinating with metal ions, and the nucleotide bases binding to the purine subsite. Across the set, ligand conformations are very similar to the crystallographically determined ATP $\alpha \mathrm{S}$ conformer, ${ }^{22}$ whereby the adenine $\mathrm{NH}_{2}$ group is located $2.99 \AA$ from the side-chain oxygen of the Asp 1018, and $3.05 \AA$ from the $\mathrm{NH}^{3+}$ of Lys 938. The ribosyl oxygens interact electrostatically with the side-chain $\mathrm{O}$ of Ser 1028 (3.86 $\AA$ distant) and the side-chain nitrogen of Asn 1025 (4.16 ̊). $\gamma$-Thiophosphate analogs were predicted to interact more strongly with receptor metal $\mathrm{B}$, and appear to form shorter H-bonds with the Gly 399 backbone $\mathrm{NH}$ and Lys 1065 side-chain $\mathrm{NH}^{3+}$ because of a longer $\mathrm{P}-\mathrm{S}$ bond length. Structural analysis suggests that phosphoamidate tails apparently do not create additional H-bonds, and also do not lead to shorter or stronger interactions between the phosphate with receptor metal ions or other residues. Consequently, it is not surprising to find that NppNHps are less potent inhibitors than NTP $\gamma$ Ss.

Our recent work also reported COMBINE analysis for the NRM species. ${ }^{18}$ While much of the analysis need not be reiterated herein, some key trends are very noteworthy with respect to their difference relative to our studies on ribosyl-modified analogs herein. The key distinction is that while residues near the ligand phosphate tail appeared to play little role in differentiating relative potency in the current study of ribosyl-substituted nucleotides, all 20 of the residues most significant to NRM nucleotide binding are clustered near the phosphate. ${ }^{18}$ This suggests that much greater NRM ligand affinity variation occurs as a function of phosphate tail modifications than from differences in the purine or pyrimidine base. As suggested earlier, other receptor features, most notably the hydrophobic pocket, appear to play the key roles in affixing ribose-substituted species and, in the case of MANT nucleotides, establishing their inhibitive superiority.

Figuring prominently among the phosphate-binding residues are the two receptor metal ions of relevance to NRM nucleotides. Metal ion interactions thus provide clear points of differentiation among the various inhibitors, regardless of whether the $\mathrm{AC}$ had been exposed to $\mathrm{Mg}^{2+}$ rich or $\mathrm{Mn}^{2+}$ rich media. It is interesting to note that metal A contributes more to VDW interactions, whereas B's contribution is more slanted toward electrostatics. The latter may be due to close coupling between metal B and all three phosphoanionic centers, whereas metal A interacts closely with only the $\alpha$-phosphate. Since some single phosphate (NMPs) species do exhibit marginal inhibition of the AC nucleotide site, metal A must still serve key roles in both VDW and electrostatic coupling.

For NRM nucleotides, an electrostatic contribution from Asp 440 also appears on the list of significant residues in both the $\mathrm{Mn}^{2+}$ and $\mathrm{Mg}^{2+}$ enriched cases. ${ }^{18}$ Since it is located closest to the ligand anionic phosphate tail, Asp 440 likely makes a negative contribution to the net binding in most cases, with its role in differentiating ligands being a question of how close the ligand is forced to approach the aspartate carboxyanion. It may be

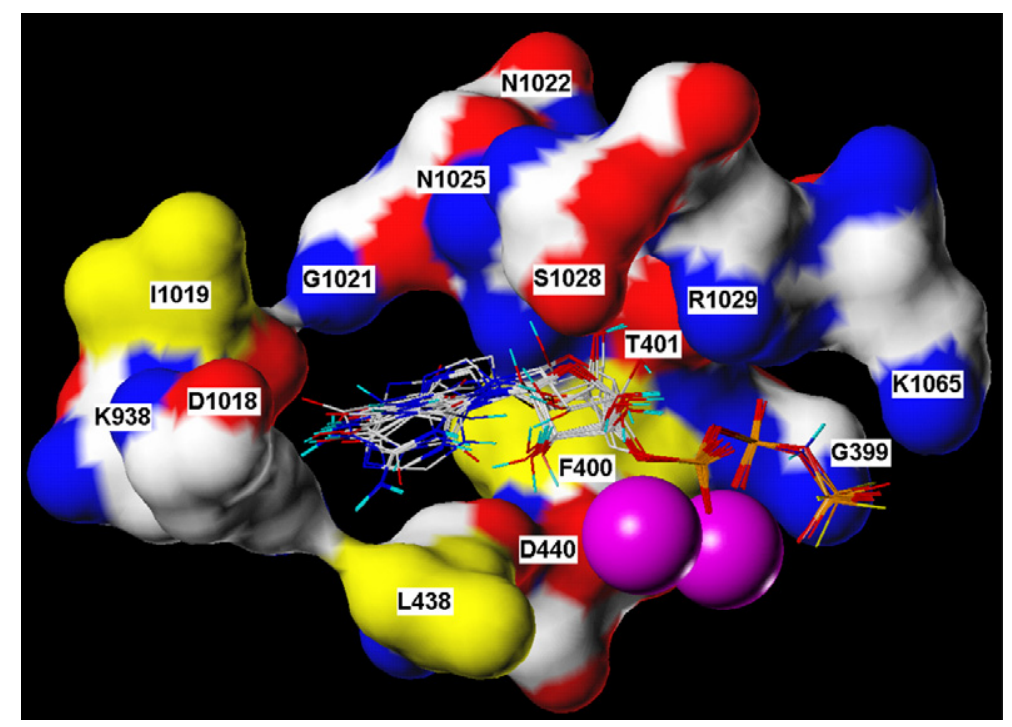

Figure 5. Computationally predicted conformers for the full training set of 17 purine and pyrimidine NRM nucleotides reported in our recent work, ${ }^{18}$ all bound within the AC active site corresponding to the $\mathrm{Mn}^{2+}$-enriched species as crystallized in conjunction with the ATP $\alpha \mathrm{S}$ ligand. Within the receptor, only selected key residues are depicted, as space-filling models colored as follows: yellow, hydrophobic surface; red, electronegative; blue, electropositive; white, polar/neutral. Ligands are depicted in stick form, with the following color scheme: red, O; gray, C; white, H; blue, N; yellow, S; orange, P. Metal ions are depicted as magenta spheres. 
possible, however, to devise H-bond donating ribosyl substituents that can take advantage of this strong acceptor.

It should be noted that among NRM species, the ribose itself does not appear to engage in strong interactions with the receptor. In the ATP $\alpha \mathrm{S}$ case, the ribose does not engage in any plausible H-bonding, although the side-chain oxygen of Ser 1028 does interact electrostatically with the $2^{\prime}$ hydroxyl, $3^{\prime}$ hydroxyl, and $5^{\prime}$ furanyl oxygens on the ribose, via distances of $4.73,3.45$, and $3.89 \AA$, respectively. A side-chain $\mathrm{N}$ on Arg 1029 is also located within $4.25 \AA$ of the $3^{\prime}$ hydroxyl.

\subsection{Receptor flexibility}

Variations in receptor conformations as a function of differing inhibition state frequently involve torsional flexibility among side chain residues in the receptor region. To some extent this occurs in the AC case in comparing the crystallographically determined binding modes of ATP $\alpha \mathrm{S}^{18}$ and MANT-GTP. ${ }^{14}$ Within the purine binding site, for example, Lys 938 plays a different role in the MANT-GTP binding than it does for ATP $\alpha$ S: the side chain $\mathrm{NH}_{3}{ }^{+}$position differs by $2.3 \AA$ between the two structures, coupling with the purine ring via nitrogen interaction on ATPaS but via oxygen on MANT-GTP. In the phosphate binding region, the Arg 1029 side-chain also has significant torsional motion around $\mathrm{C} \gamma-\mathrm{C}_{\delta}$ bond, with the position of terminal nitrogens differing by about $6.1 \AA$ from one structure to the other, forming an H-bond to the phosphate of ATP $\alpha$ S, but not for MANT-GTP. To check the energy cost for such movement in Arg 1029, a dihedral energy plot was calculated and is shown in Figure 6. In the AC/MANT-GTP crystal structure, the torsion is in one low energy region around $-66.2^{\circ}$, whereas the value for the ATPaS bound structure is within another low energy region located between $130^{\circ}$ and $180^{\circ}$.

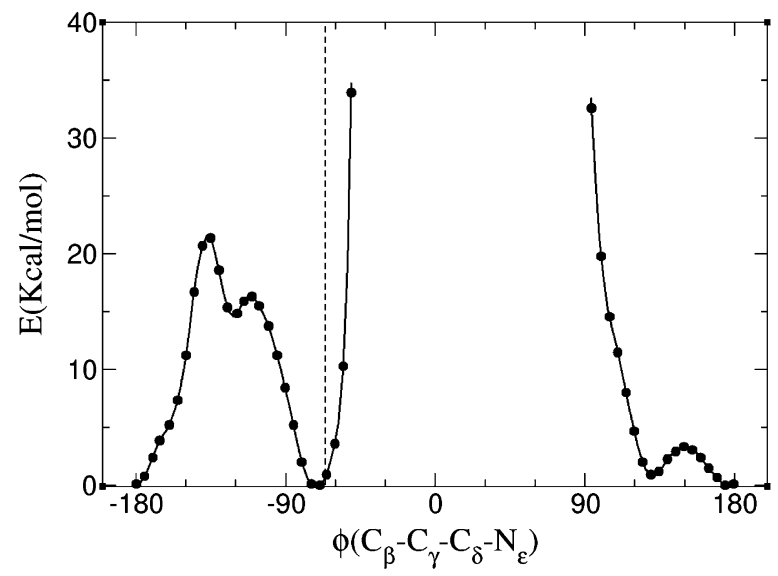

Figure 6. The dihedral energy plot for the $\mathrm{C}_{\beta}-\mathrm{C}_{\gamma}-\mathrm{C}_{\delta}-\mathrm{N}_{\varepsilon}$ torsion angle of Arg 1029. The dashed vertical line indicates the crystallographically observed value $\left(-66.2^{\circ}\right)$. The high barrier from $-50^{\circ}$ to $100^{\circ}$ reflects a direct clash with Ser 1032, Thr 1033, and nearby residues, whereas the small barrier from $-180^{\circ}$ to $-66^{\circ}$ indicates weaker VDW interaction with Asn 1063, Val 1064, and Lys 1065. The energy was calculated in the AC crystal structure corresponding to the bound MANT-GTP case, but with MANT-GTP removed.
It is also apparent from the two crystal structures that the side-chain of Asn 1025 has a different conformation in the presence of ATPaS than it does for the MANTGTP bound conformation, and that the latter will permit favorable interaction with the oxygen in the position $3^{\prime}$ of the ribose ring, as was consistent with our analysis of ribose-substituted nucleotides binding to the receptor in the MANT-GTP bound conformation. This Asn 1025 flexibility suggests a possible avenue for further optimization of MANT inhibitors: Replacing the MANT methyl group with a longer H-bond acceptor (e.g., $\mathrm{CH}_{2} \mathrm{OH}$ ) might increase the affinity by permitting interactions with the Asn 1022 side chain $\mathrm{NH}_{2}$ (currently $4.05 \AA$ from the methyl).

It would appear that not all of the differences between the crystal structures of MANT-GTP inhibited AC and the ATPaS bound system arise from side-chain relaxations, however. While the all-atom RMSD values between the two superimposed structures are $1.23 \AA$ for chain $\mathrm{A}$ and $1.11 \mathrm{~A}$ for chain $\mathrm{B}$, the net deviations are quite similar when comparing $\mathrm{C} \alpha$ atoms $(0.90 \AA$ for chain $\mathrm{A}$ and $0.70 \AA$ for $\mathrm{B}$ ) and the corresponding backbone atoms (0.92 and $0.72 \AA$, respectively). This is tangible evidence of the subtle but non-trivial structural dependence on AC ligation state that has been suggested previously. ${ }^{7,14,22,23}$ It also suggests that any serious attempt to design optimal AC inhibitors should explicitly consider these differences.

From crystallographic analysis, it is apparent that the MANT-GTP bound AC has a conformation somewhere between the open and closed states. ${ }^{14}$ The most obvious structural difference of this system relative to that of NRM inhibitors such as ATPaS is in the MANT binding region. In the ATPaS bound conformation, the positions of Asn 1025 (both main and side-chains) and the phenyl ring of the Phe 400 collectively form a narrow gorge with closest inter-atomic spacing of $3.36 \AA$. In this case, a strong steric hindrance would clearly prevent groups such as the MANT from binding. In the MANT-GTP crystal structure, however, the spacing is no less than $7.06 \AA$ throughout, which constitutes sufficient space for MANT entry into the pocket.

To further verify the influence of the conformation change of the AC on the binding affinity of ribosesubstituted and NRM nucleotides, we attempted to (a) dock MANT-substituted nucleotides into the receptor from the AC/ATP $\alpha$ S crystal structure, and (b) dock NRM nucleotides into the receptor from the $\mathrm{ACl}$ MANT-GTP structure. In the first case, the computations all failed. This indicates that without some form of receptor conditioning (as was performed in a prior study using molecular dynamics simulations), ${ }^{16}$ the receptor structure suitable for binding NRM species is not conducive to MANT-ligand binding, largely as a result of steric hindrance arising from Asn 1025 and Phe 400. In the second case, the NRM nucleotides did successfully bind within the receptor. However an ensuing COMBINE analysis yielded poor correlation relative to experimentally determined assay data. Given the high quality COMBINE correlation achieved for 
these ligands with respect to the AC/ATP $\alpha \mathrm{S}$ case (i.e., $R^{2}=0.93, Q^{2}(\mathrm{LOO})=0.71$ for excess $\mathrm{Mn}^{2+} ; R^{2}=0.89$, $Q^{2}=0.75$ for $\left.\mathrm{Mg}^{2+}\right),{ }^{18}$ it appears fairly certain that the latter failure is also due to differences in AC receptor structure between the two models. It is our assertion that a strong lipophilic affinity between the MANT group and the hydrophobic pocket is capable of inducing the conformational differences observed in comparing the two structures.

\section{Conclusions}

The COMBINE methodology provides an excellent strategy for performing detailed structure-activity relationship analysis for targets where both experimental inhibition data and a receptor crystal structure are available. In this study, we have employed COMBINE analysis to derive consistent conformational predictions for a variety of ribose-substituted nucleotides bound to the AC nucleotide binding site, achieving a model with strong correlation relative to experiment $\left(R^{2}=0.89\right)$ and reasonable cross-validated predictivity $\left(Q^{2}=0.61\right)$. To augment prior SAR analyses based largely on crystal structure inspection and low-level modeling studies, our COMBINE model highlights a number of residues that may serve key roles in differentiating the relative ligand affinity and inhibitive potential. In the triphosphate binding site, such residues include Thr 401, whose backbone NH provides an important H-donor site for the phosphate tail, and Ile 397 and Ala 385, both of which are considered to be unfavorable sites for van der Waals contact. In the purine binding pocket, residue importance is dictated largely by electrostatics, with the backbone carbonyl providing a potential beneficial $\mathrm{H}$-acceptor site, and the backbone amide protons of Gly 439 and Val 1024 offering prospective H-donation targets. Our COMBINE analysis was not effective in resolving pharmacophoric trends within the hydrophobic pocket, largely due to non-conservation of binding modes by lipophilic ribose substituents. This may be ameliorated given access to more experimental training data for set of ligands with structural conservation in the triphosphate and ribose moieties and greater diversity among ribose substituents.

For ligands with the capacity for low-barrier interconversion between $2^{\prime}$ - and $3^{\prime}$-ribose substituents, the COMBINE model training afforded systematic evaluation of the comparison of the two isomeric forms, and predicted that the majority of ligands in our training set should adopt the $3^{\prime}$-substitution form, in contrast with prior modeling work, ${ }^{16}$ but corresponding well with recent crystallographic evidence for MANTGTP. ${ }^{14}$ Comparing the manifold of predicted ligand conformers of these ribose-modified ligands versus a prior manifold of NRM nucleotides ${ }^{18}$ and the crystal structures derived from $\mathrm{ATP} \alpha \mathrm{S}$-inhibitor $\mathrm{AC}$ versus the analogous MANT-GTP structure has allowed us to posit that a specific receptor relaxation occurs, primarily involving an alternative conformation of the Arg 1029 side-chain, that differentially favors the $3^{\prime}$-substitution form.

\section{Methodology}

The receptor structure model used herein for evaluating AC inhibition by ribosyl-substituted nucleotides was adapted from an X-ray crystal structure for AC in complex with MANT-GTP (PDB ID: 1TL7). ${ }^{14}$ This is in contrast to our previous work on non-ribosyl-modified (NRM) nucleotides, in which we employed a receptor model derived from an AC/ATP $\alpha \mathrm{S}$ crystal structure. ${ }^{18}$ The receptor model was prepared by eliminating all residues located more than $15.0 \AA$ from the position of the co-crystallized MANT-GTP ligand, leaving a total of 120 residues within the receptor model (a full list, including relevant coefficients, is provided in the supporting information). The ligand itself was then also eliminated, and the remaining structure was protonated to yield ionic aspartate, glutamate, lysine, and arginine residues, and all other atoms saturated to neutrality. Unrealistic atomic contacts were alleviated by energetically minimizing all hydrogen atom coordinates (heavy atoms spatially fixed) via the AMBER '89 force field and corresponding charges, ${ }^{24}$ as implemented in the MOE software suite. ${ }^{25}$ The AC receptor contains two metal ions that, under normal physiological conditions, include one $\mathrm{Mg}^{2+}$ and one $\mathrm{Mn}^{2+}$. The nature of these ions has been identified as having a major effect on ligand binding affinity ${ }^{14-16,18}$ and thus separate receptor models were constructed for the normal $\left(\mathrm{Mg}^{2+}+\mathrm{Mn}^{2+}\right)$, magnesium enriched $\left(\mathrm{Mg}^{2+}+\mathrm{Mg}^{2+}\right)$, and manganese enriched $\left(\mathrm{Mn}^{2+}+\mathrm{Mn}^{2+}\right)$ species for which assay data exist. As crystal structures have elucidated little impact on the surrounding protein structure as a function of receptor ion exchange, ${ }^{22}$ a structure of $\mathrm{Mn}^{2+}$ enriched AC was obtained by simply substituting the native $\mathrm{Mg}^{2+}$ receptor metal ion for a second $\mathrm{Mn}^{2+}$ ion. The $\mathrm{Mg}^{2+}$ enriched model was obtained in an analogous fashion. It should be noted that such a truncated model (i.e., with the $15.0 \AA$ cutoff for residue retention), while computationally expeditious, does preclude the possibility of rigorous examination of receptor relaxation response effects. We intend to address these effects in future work.

The collection of ligands examined in this study focused primarily on nucleotide analogs, most with assorted ribosyl modifications, for which experimental AC inhibition data were available. Specifically, the manifold included standard mono-, di-, and triphosphate (i.e., NMP, NDP, and NTP) nucleotides, plus some $\gamma$-thio analogs thereof (i.e., NTP $\gamma$ S), and others with amino linkers between the $\beta$ and $\gamma$ phosphates (i.e., NppNHp). Ribosyl modifications include phosphate cyclization (e.g., cAMP), unadorned 2'-deoxy nucleotide analogs (e.g., 2'-d-UTP), and oxy-substituents including dipyrromethene boron difluoride (BODIPY), MANT and its $n$-hexyl (MAH) analog. The full set of ligands considered herein, along with observed experimental $K_{\mathrm{i}}$ data in conjunction with inhibition constants (for both normal and $\mathrm{Mn}^{2+}$-enriched $\mathrm{AC}$ ) obtained from our previous work, ${ }^{16}$ is reported in Table 1 .

Structures for the ligands listed in Table 1 were prepared via SYBYL ${ }^{26}$ and optimized using the Tripos 
Force Field ${ }^{27}$ and Gasteiger-Marsili electrostatics ${ }^{28}$ as implemented in SYBYL 7.2, ${ }^{26}$ taking account all non-bonding forces to within an $8.0 \AA$ cutoff. Other energetic and convergence settings were set to default values. All ligands were assumed to possess a fully ionized phosphate tail, with the following net charges: triphosphate $=-4$, diphosphate $=-3$, monophosphate $=-2$. All other atoms were protonated to neutrality.

Ligand-receptor complex structures were predicted via Lamarkian genetic algorithms flexible binding conformational searches as implemented in AutoDock. ${ }^{29} 100$ poses were requested for each ligand, of which only those that exhibited binding between the phosphate tail and the receptor metal cations were retained for subsequent consideration. For structure-activity modeling, the preliminary criterion for choosing a given ligand pose was a ranking by AutoDock affinity score. As is described in the subsequent paragraph, however, the original pose choice was iteratively refined according to capacity for optimizing the structure-activity partial least squares (PLS) fit.

For each complex, pairwise electrostatic and van der Waals (VDW) terms were computed between the ligand and each residue within the $15.0 \AA$ radius receptor model. These terms were evaluated according to the MMFF94 VDW parametrization, ${ }^{30}$ and electrostatics derived from MMFF94 ligand atomic charges ${ }^{30}$ (as implemented in SYBYL 7.1 $)^{26}$ and AMBER receptor charges, ${ }^{24}$ all performed via the computer program COMBSCORE. This program was developed in house, encoding all electrostatic and VDW terms in the precise form prescribed in the original MMFF94 publication. ${ }^{30}$ COMBSCORE test cases were validated relative to VDW and electrostatic energies computed in SYBYL. ${ }^{26}$ The program source code and relevant sample input files are provided in supporting information. Building on a model from our earlier study ${ }^{31}$ and functionally adapted from a prior project, ${ }^{31}$ the binding affinity for each ligand was then trained as a PLS fit of desolvation terms and the above enthalpic interactions (without any form of variable rescaling) collectively expressed via the following equation:

$$
\begin{aligned}
\mathrm{p} K_{\mathrm{i}}= & 9-\log _{10}\left(K_{\mathrm{i}}\right) \\
= & c_{\mathrm{P}}(\Delta P S A)+c_{\mathrm{N}}(\Delta N P S A) \\
& +\sum_{j}\left(c_{\mathrm{e}}(j) E_{j}^{\mathrm{ele}}+c_{\mathrm{v}}(j) E_{j}^{\mathrm{vdw}}\right)+K
\end{aligned}
$$

as based on the comparative binding energy (COMBINE) type methodology. ${ }^{32}$ The first two terms in this expression, $\triangle P S A$ (the change in the ligand's solvent accessible polar surface area upon complexation) and $\triangle N P S A$ (the corresponding change in non-polar surface area), were chosen to reflect ligand desolvation effects. $E_{i}^{\text {ele }}$ and $E_{i}^{\mathrm{vdw}}$ are electrostatic and VDW interaction enthalpies arising between the $i$ th residue and the docked ligand. Finally, the regression coefficients, $c_{\mathrm{P}}$, $c_{\mathrm{N}}, c_{\mathrm{e}}$, and $c_{\mathrm{v}}$ (for $\triangle P S A, \triangle N P S A, E_{i}^{\mathrm{ele}}$, and $E_{i}^{\mathrm{vdw}}$, respectively), were computed via the Simca- $\mathrm{P}^{33}$ program, and
$K$ is a scaling constant generated from this regression analysis.

As indicated previously, we iteratively refined the above COMBINE model by identifying ligands whose initial $\mathrm{p} K_{\mathrm{i}}$ prediction deviated by more than $25 \%$ from the experimental value, and replacing the initially selected pose with the highest ranked structure for which a deviation of less than $25 \%$ relative to experiment was possible. Note that the new conformer was only retained in the model if it yielded an improved cross-validated $\left(Q^{2}\right)$ correlation relative to experiment. In the event that no pose was obtained satisfying these requirements, the ligand was assumed to be an outlier and was omitted from the model.

Since ribosyl-modified nucleotides are susceptible to low-barrier interconversion between the $2^{\prime}$ - and $3^{\prime}$-substituent form,${ }^{34}$ our COMBINE analysis was applied in a similar manner to predict which of the two isomers was the most probable AC inhibitor. To accomplish this, an initial PLS model was computed wherein both the $2^{\prime}$ - and $3^{\prime}$-substituted versions of each nucleotide (except for $2^{\prime}$-deoxy ligands, where only the $3^{\prime}$-substitution is possible) were included. In each case, the criteria of lower relative deviations between experiment and theory, and improved $Q^{2}$ performance were used to identify the more internally consistent structure. This structure was retained in the model and its poorer performing isomer was then discarded.

For our analysis of receptor relaxation effects, torsional energies for the side-chains of select receptor residues of interest were analyzed by clearing the receptor model of all ligands and crystallographic waters, but retaining in situ metal ions, as the latter are considered vital to receptor function. The total potential energy of the system was then evaluated using MOE with AMBER force field and charges for all conformers of the target torsional degree of freedom along a $360^{\circ}$ rotation with a $5^{\circ}$ increment. All other bond lengths, angles, and dihedrals were fixed during such searches.

\section{Acknowledgments}

The authors thank Andreas Gille (Department of Pharmacology and Toxicology, University of Kansas) for stimulating discussions. This work was supported by grants of the Heartland Affiliate of the American Heart Association (0450120Z), the Deutsche Forschungsgemeinschaft (SE 529/5-1) (R.S.), NIH Grant No. P20 RR016475 from the INBRE Program of the National Center for Research Resources (G.H.L.), and by computer time from the National Computational Science Alliance (MCB03001N) (J.G.).

\section{Supplementary data}

Supplementary data associated with this article can be found, in the online version, at doi:10.1016/j.bmc. 2007.02.014. 


\section{References and notes}

1. Tang, W. J.; Hurley, J. H. Mol. Pharmacol. 1998, 54, 231-240.

2. Sunahara, R. K.; Dessauer, C. W.; Gilman, A. G. Аnпи. Rev. Pharmacol. Toxicol. 1996, 36, 461-480.

3. Bohm, M. Mol. Cell. Biochem. 1995, 147, 147-160.

4. Okumura, S.; Takagi, G.; Kawabe, J.; Yang, G.; Lee, M. C.; Hong, C.; Liu, J.; Vatner, D. E.; Sadoshima, J.; Vatner, S. F.; Ishikawa, Y. Proc. Natl. Acad. Sci. U.S.A. 2003, 100, 9986-9990.

5. Di Marzo, V.; Melck, D.; De Petrocellis, L.; Bisogno, T. Prostaglandins Other Lipid Mediat. 2000, 61, 43-61.

6. Roitman, G.; Levine, J.; Bersudsky, Y.; Belmaker, R. H. Human Psychopharmacol.: Clin. Exp. 1998, 13, 121-125.

7. Tesmer, J. J. Nat. Struct. Mol. Biol. 2005, 12, 7-8.

8. Esposito, G.; Jaiswal, B. S.; Xie, F.; Krajnc-Franken, M. A.; Robben, T. J.; Strik, A. M.; Kuil, C.; Philipsen, R. L.; van Duin, M.; Conti, M.; Gossen, J. A. Proc. Natl. Acad. Sci. U.S.A. 2004, 101, 2993-2998.

9. Tesmer, J. J.; Sunahara, R. K.; Gilman, A. G.; Sprang, S. R. Science 1997, 278, 1907-1916.

10. Tang, W. J.; Stanzel, M.; Gilman, A. G. Biochemistry 1995, 34, 14563-14572.

11. Yan, S. Z.; Huang, Z. H.; Shaw, R. S.; Tang, W. J. J. Biol. Chem. 1997, 272, 12342-12349.

12. Zhang, G.; Liu, Y.; Ruoho, A. E.; Hurley, J. H. Nature 1997, 386, 247-253.

13. Tesmer, J. J.; Dessauer, C. W.; Sunahara, R. K.; Murray, L. D.; Johnson, R. A.; Gilman, A. G.; Sprang, S. R. Biochemistry 2000, 39, 14464-14471.

14. Mou, T. C.; Gille, A.; Fancy, D. A.; Seifert, R.; Sprang, S. R. J. Biol. Chem. 2005, 280, 7253-7261.

15. Gille, A.; Seifert, R. J. Biol. Chem. 2003, 278, 12672-12679.

16. Gille, A.; Lushington, G. H.; Mou, T. C.; Doughty, M. B.; Johnson, R. A.; Seifert, R. J. Biol. Chem. 2004, 279, 19955-19969.
17. Mou, T.-C.; Suryanarayana, A. G. S.; Richter, M.; Seifert, R.; Sprang, S. R. Mol. Pharmacol. 2006, 70, 878-886.

18. Gille, A.; Guo, J.-X.; Mou, T.-C.; Doughty, M. B.; Lushington, G. H.; Seifert, R. Biochem. Pharmacol. 2005, 71, 89-97.

19. Mou, T. C.; Gille, A.; Suryanarayana, S.; Richter, M.; Seifert, R.; Sprang, S. R. Mol. Pharmacol. 2006, 70, 878886.

20. Bruna, P. J. M.; Robert, C.; Grein; Friedrich Int. J. Quantum Chem. 1995, 29, 455-463.

21. Gille, A.; Liu, H. Y.; Sprang, S. R.; Seifert, R. J. Biol. Chem. 2002, 277, 34434-34442.

22. Tesmer, J. J.; Sunahara, R. K.; Johnson, R. A.; Gosselin, G.; Gilman, A. G.; Sprang, S. R. Science 1999, 285, 756760 .

23. Hurley, J. H. J. Biol. Chem. 1999, 274, 7599-7602.

24. Weiner, S. J.; Kollman, P. A.; Nguyen, D. T.; Case, D. A. J. Comp. Chem. 1986, 7, 230-252.

25. Molecular Opperating Enviroment (MOE) 2002.03, Chemical Computing Group Inc.: Mar, 2002.

26. SYBYL 7.2, The Tripos Associates, Inc.: 2006.

27. Clark, M.; Cramer, R. D., III; Van Opdenbosch, N. J. Comput. Chem. 1989, 10, 982-1012.

28. Gasteiger, J.; Marsili, M. Tetrahedron 1980, 36, 32193228.

29. Morris, G. M.; Goodsell, D. S.; Halliday, R. S.; Huey, R.; Hart, W. E.; Belew, R. K.; Olson, A. J. J. Comput. Chem. 1998, 19, 1639-1662.

30. Halgren, T. A. J. Comput. Chem. 1999, 20, 720-729.

31. Guo, J.; Hurley, M. M.; Wright, J. B.; Lushington, G. H. J. Med. Chem. 2004, 47, 5492-5500.

32. Ortiz, A. R.; Pisabarro, M. T.; Gago, F.; Wade, R. C. J. Med. Chem. 1995, 38, 2681-2691.

33. SIMCA-P, Umereics AB: July, 2001.

34. Jameson, D. M.; Eccleston, J. F. Methods Enzymol. 1997, 278, 363-390. 